\title{
MCSS-based Predictions of Binding Mode and Selectivity of Nucleotide Ligands
}

\author{
Roy González-Alemán, ${ }^{\dagger} \ddagger$ Nicolas Chevrollier, ${ }^{\dagger}$ Manuel Simoes, $₫$ Luis \\ Montero-Cabrera, ${ }^{\ddagger}$ and Fabrice Leclerc ${ }^{*} \dagger$ \\ $\dagger$ Institute for Integrative Biology of the Cell (I2BC), CEA, CNRS, Université Paris Saclay, \\ Gif-sur-Yvette, F-91198, France \\ $\ddagger$ Laboratorio de Química Computacional y Teórica (LQCT), Facultad de Química, \\ Universidad de La Habana, 10400 La Habana, Cuba \\ ฯCPC Manufacturing Analytics, Strasbourg, France \\ E-mail: fabrice.leclerc@i2bc.paris-saclay.fr \\ Phone: +33 (0)1 69826239
}

\section{Contents}

Supporting Information Available

MCSS

Scoring 


\section{Supporting Information Available}

\section{Benchmark of 121 protein-nucleotide complexes}

1. Attached Supplementary Data 1 (Data-S1.csv): a list of PDB IDs including the ligand ID, the atomic resolution, functional classification, and EC number.

2. Attached Supplementary Data 2 (Data-S2.csv): calculations of the BINANA features (number of contacts, number of H-bonds, the buried fraction of ligand, etc)

3. Attached Supplementary Data 3 (Data-S3.csv): calculations of the NACCESS surface terms for the fraction of buried surface of the ligand

4. Attached Supplementary Data 4 (Data-S4.tar.gz): 2D diagrams of the contacts within the binding sites (SVG format). 

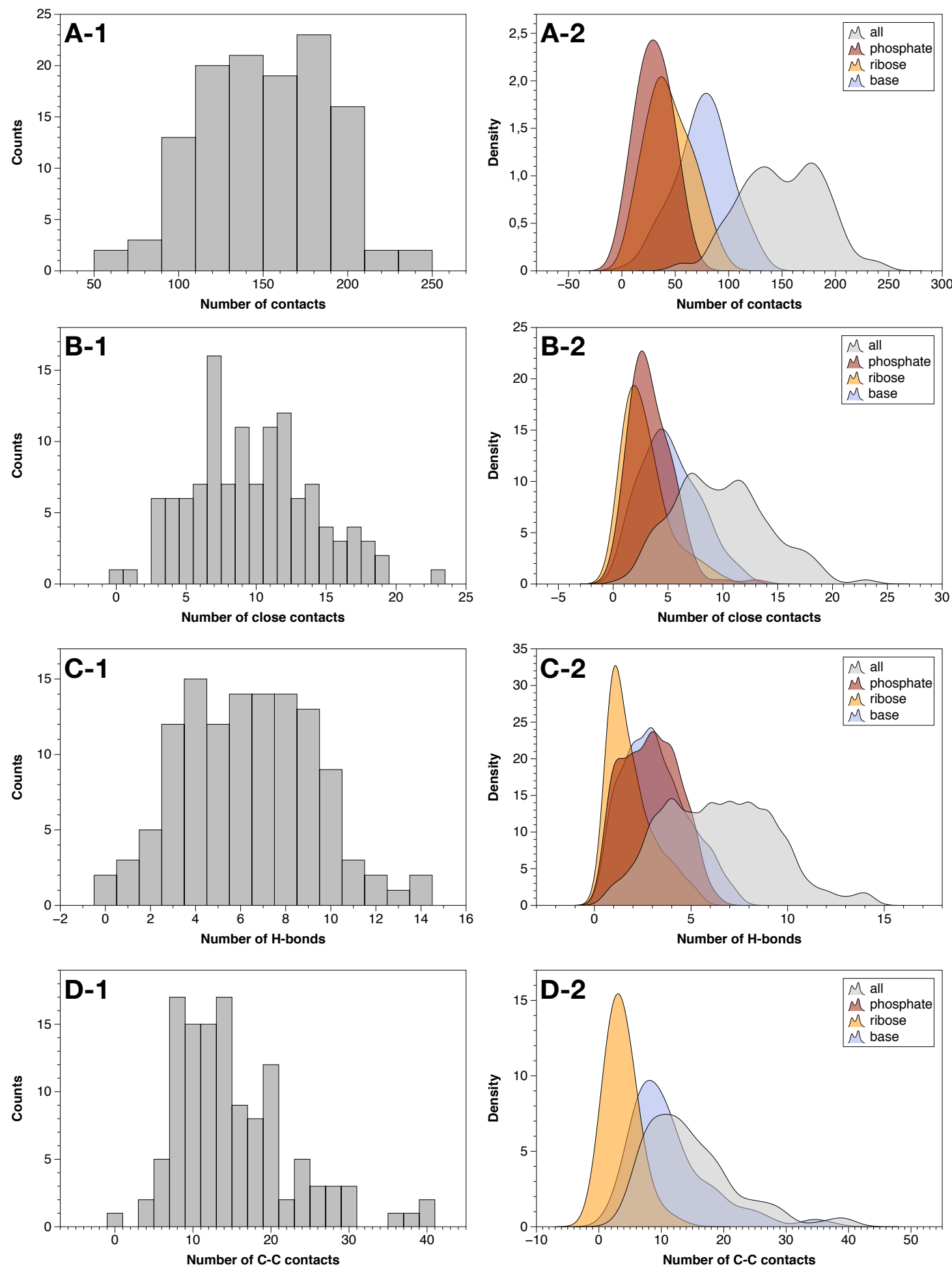

Supplementary Figure 1: Molecular and energy features of the nucleotide-binding sites from the benchmark of 121 complexes. A-1.: Histogram of the number of contacts; A-2: Smooth histogram with decomposition per nucleotide moiety (base, ribose, phosphate); B-1.: Histogram of the number of close contacts; B-2.: Same as A-2 for close contacts; C-1.: Histogram of the number of H-bonds; C-2.: Same as A-2 for H-bonds; D-1.: Histogram of the number of C-C contacts; D-2.: Same as A-2 for C-C contacts; (to be continued). 

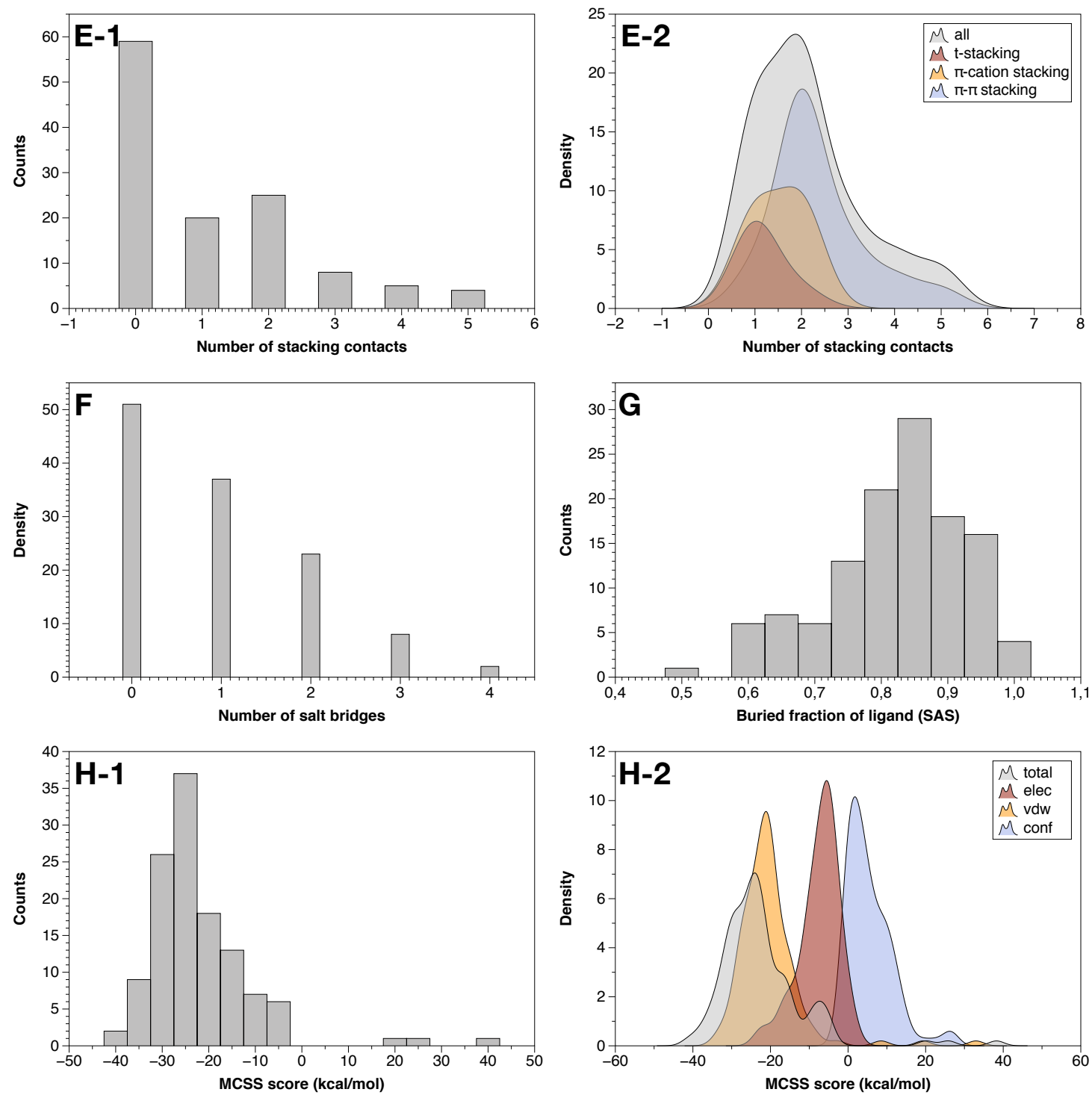

Supplementary Figure 1: Molecular and energy features of the nucleotide-binding sites from the benchmark of 121 complexes (continued). E-1.: Histogram of the number of stacking contacts; E-2.: Smooth histogram with decomposition per stacking types; F.: Histogram of the number of salt-bridges; G.: Histogram of the buried fraction of ligand (calculated from the solvent accessible surface); H-1.: Histogram of the MCSS scores calculated for the ligands optimized in their binding site; H-2.: Smooth histogram with decomposition per contribution types (electrostatics, van der Waals, conformational). The molecular descriptors associated with the atomic contacts are calculated by BINANA; ${ }^{1}$ the stacking contributions are calculated from OpenEye; ${ }^{2}$ the MCSS score is calculated by the scoring function derived previously. ${ }^{3}$ 

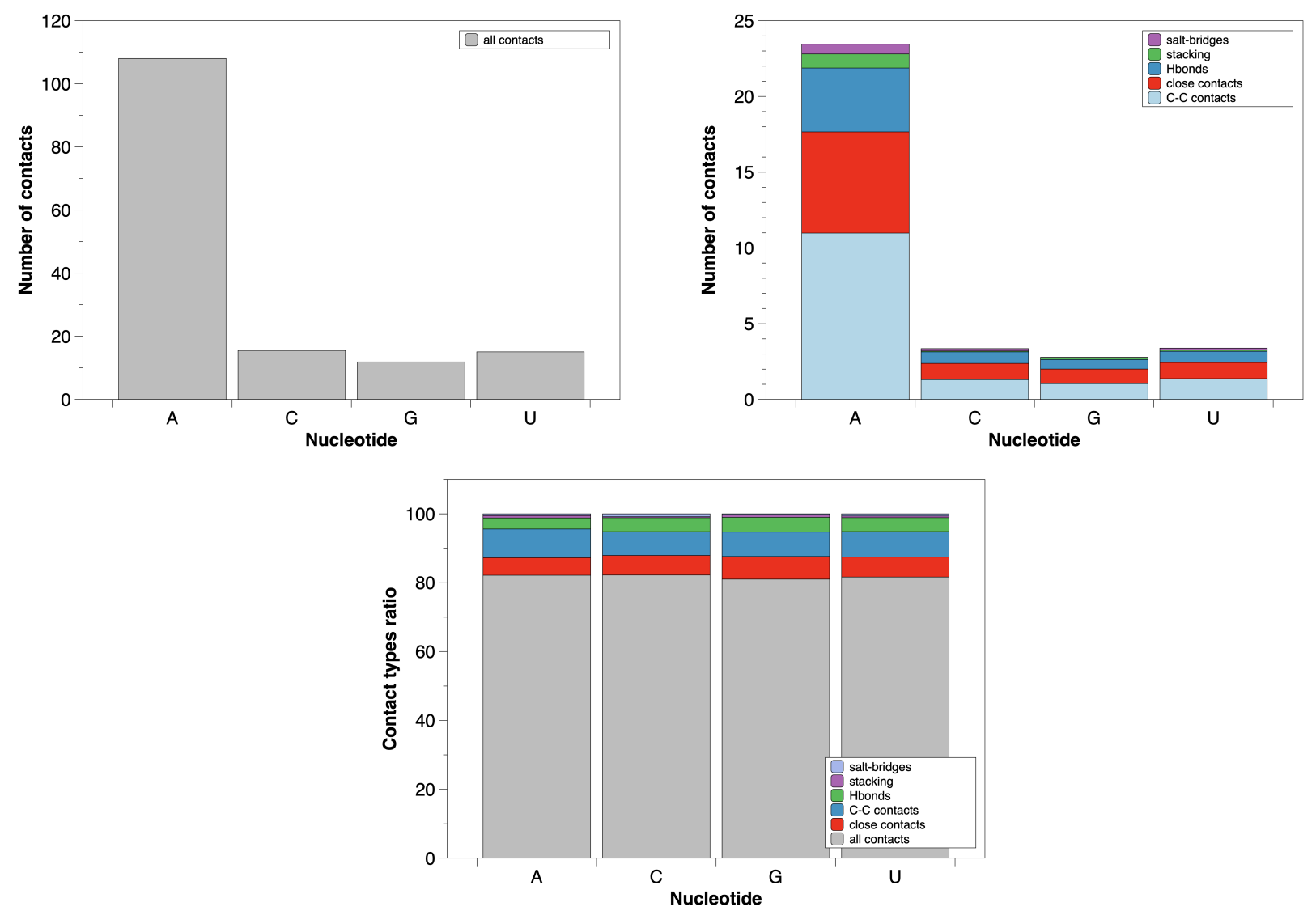

Supplementary Figure 2: Nucleotide breakdown of atomic contacts. Top-left: all contacts; top-right: specific contacts (C-C contacts, close contacts, Hbonds, stacking contacts, saltbridges); bottom: ratio of each type of specific contacts. The number of contacts correspond to the average value over the full benchmark. 

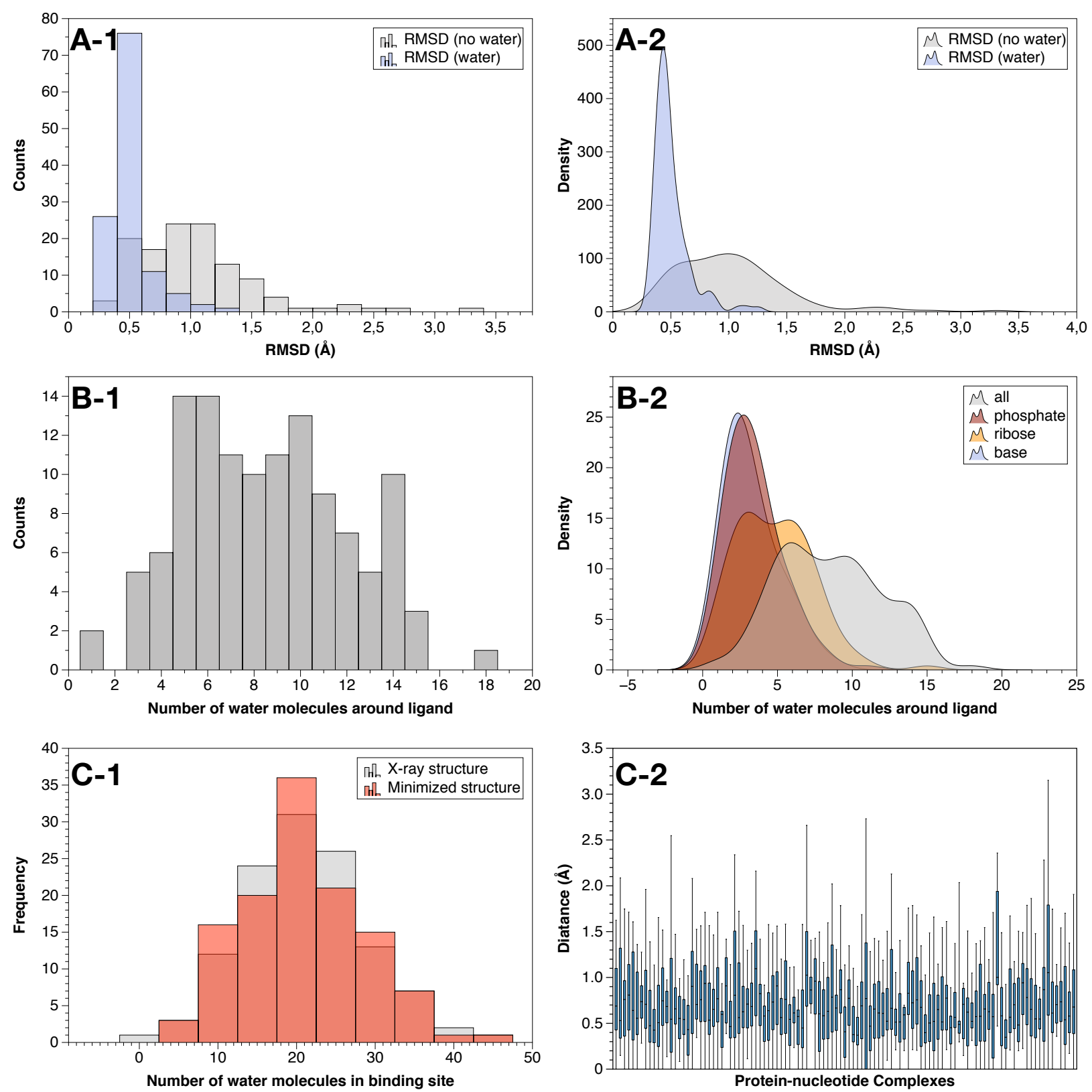

Supplementary Figure 3: Distributions of water molecules and impact on the binding sites. A-1.: Histogram of RMSD in presence/absence of water molecules; A-2.: Same as A-1 with a smooth histogram; B-1.: the number of water molecules around the ligand (distance cutoff of 4.0 $\AA$ ); B-2.: Same as B-1 with decomposition per nucleotide moiety; C-1.: Number of water molecules within the binding site as defined in MCSS by the box parameters (see Methods); C-2.: displacements $(\AA)$ of water molecules from their crystallized positions. 


\section{MCSS}

5. Attached Supplementary Data 5: MCSS input sample (Data-S5.txt)

6. Attached Supplementary Data 6: MCSS nonbonded parameters sample (Data-S6.txt)

7. Attached Supplementary Data 7 (Data-S7.csv): MCSS score (including its VdW and elec terms) and RMSD values for each protein-nucleotide complex 

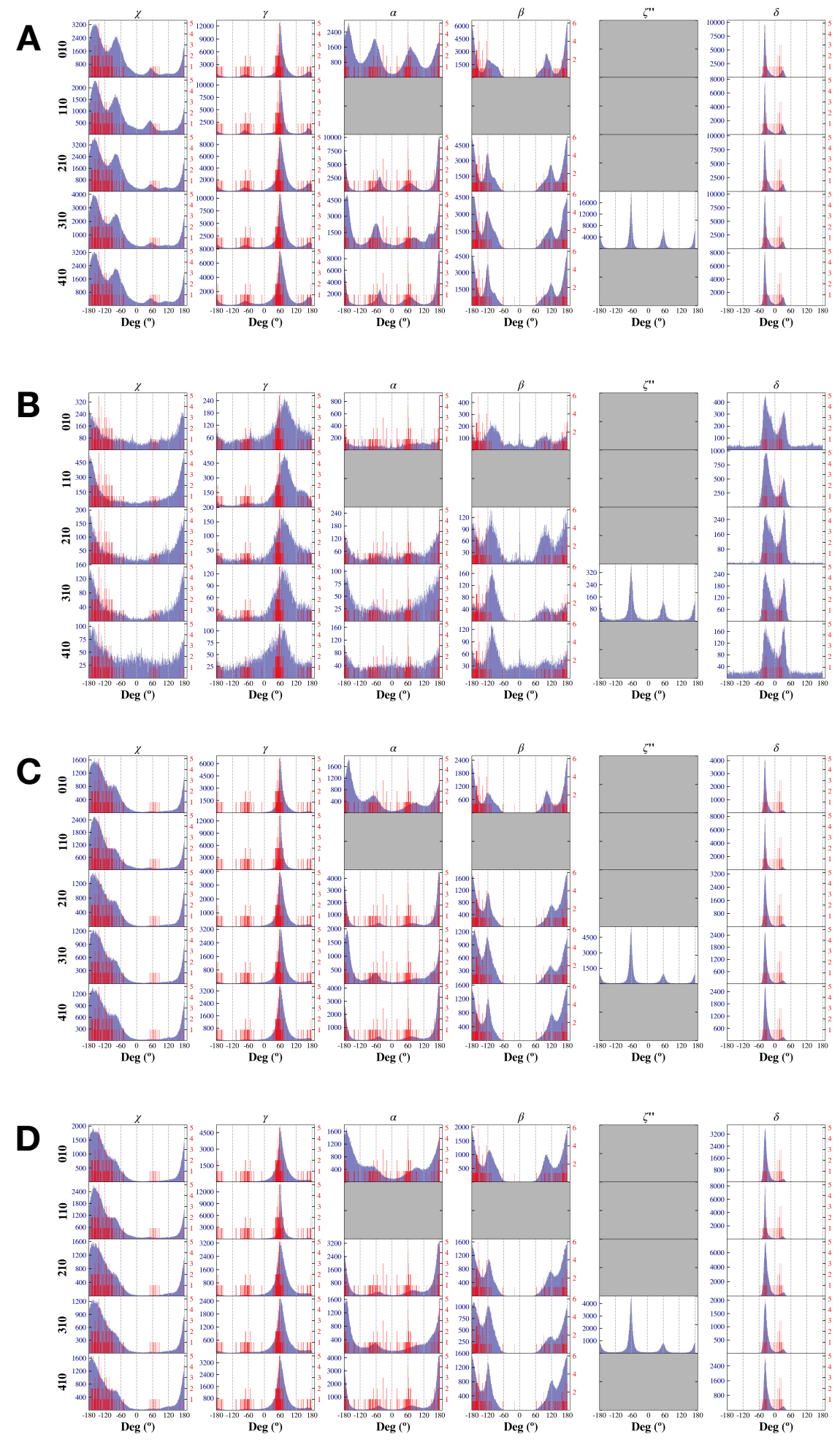

Supplementary Figure 4: Torsions angles. Nonbonded models and associated patches (R010 to R410): A. SCAL, B. FULLW, C. SCALW, D. STDW. In blue: the distribution of the torsions angles observed in the MCSS minima; In red: the distribution of the torsions angles observed in the bound ligands. 


\section{Scoring}

Autodock Vina is a well-known docking method used for virtual screening; the associated scoring function is pretty robust, having regularly been used in the comparative assessment of scoring functions (CASF) challenges. ${ }^{4}$ Vinardo and $\Delta_{\text {vina }} R F_{20}$ were both derived from Vina and tested in the CASF-2013 challenge. Vinardo was optimized and validated on large datasets. ${ }^{5}$ It was tested in particular on the DUD library that contains, among other proteins, kinases with nucleotide ligands or nucleotide analogs. ${ }^{6}$ $\Delta_{\text {vina }} R F_{20}$ was derived more recently from Vina with a new parametrization based on random forest. The performance of $\Delta_{v i n a} R F_{20}$ was superior to that of Vina when tested on the CASF-2007 and CASF-2013 challenges benchmarks. Finally, ITscorePR was included since it has been specifically developed for protein-RNA interactions. The scores calculated with all the scoring functions: ITscorePR, ${ }^{7} \Delta_{\text {vina }} R F_{20},{ }^{8}$ Autodock Vina score,${ }^{9}$ Vinardo, ${ }^{5}$ and the MM-GB models (see Methods) except MCSS $^{3}$ correspond to single-point calculations on the MCSS-generated poses.

8. Attached Supplementary Data 8 (Data-S8.tar.gz): selectivity diagrams SCAL/STDW for the native poses for each protein-nucleotide complex of the benchmark. 


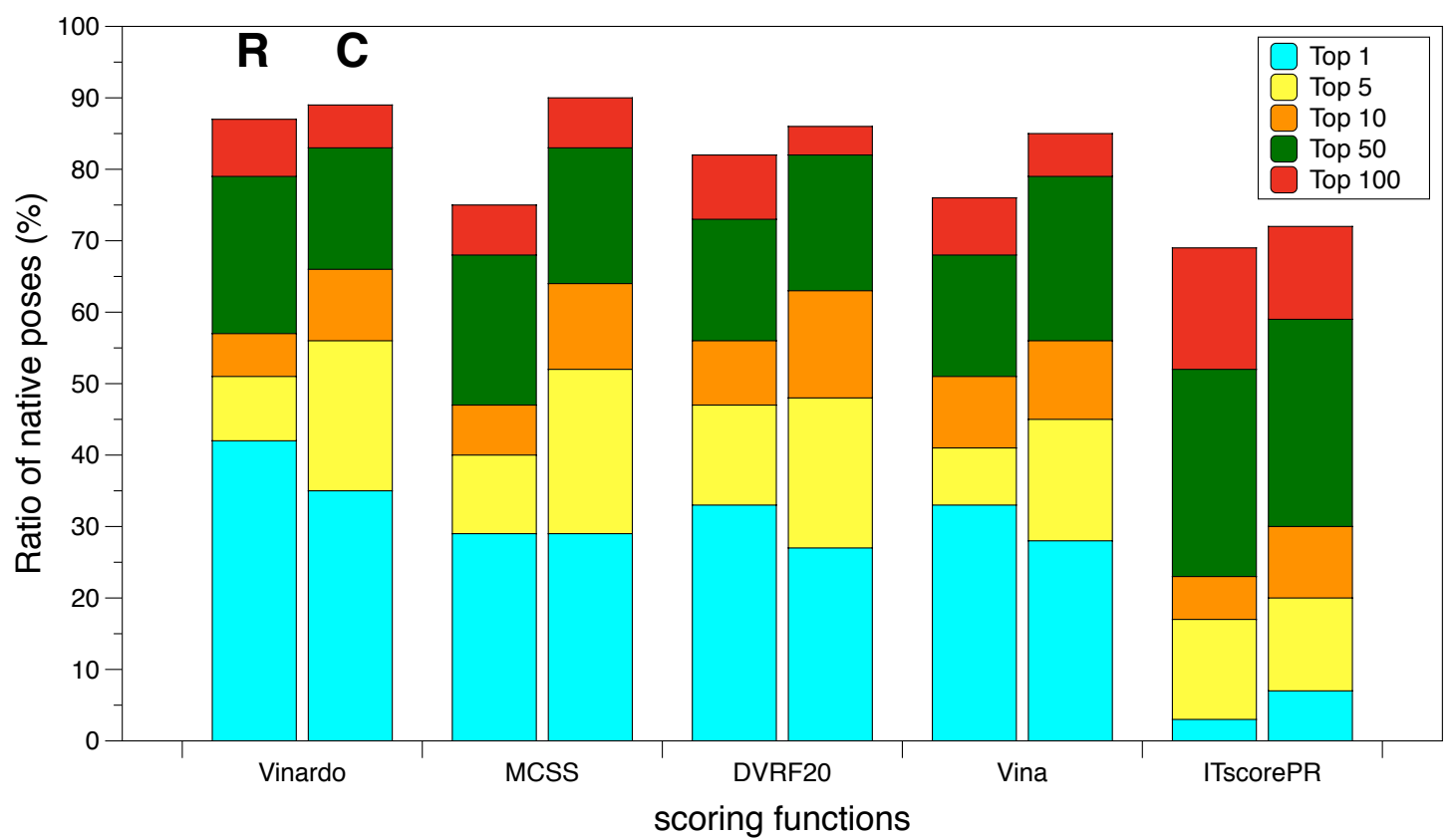

Supplementary Figure 5: Docking powers (top1 to top100) for Vinardo, MCSS, $\Delta_{\text {vina }} R F_{20}$, Vina, and ITscorePR and the impact of the clustering filtering (using the patch R310). Left bar (R): no clustering; Right bar (C): clustering. 

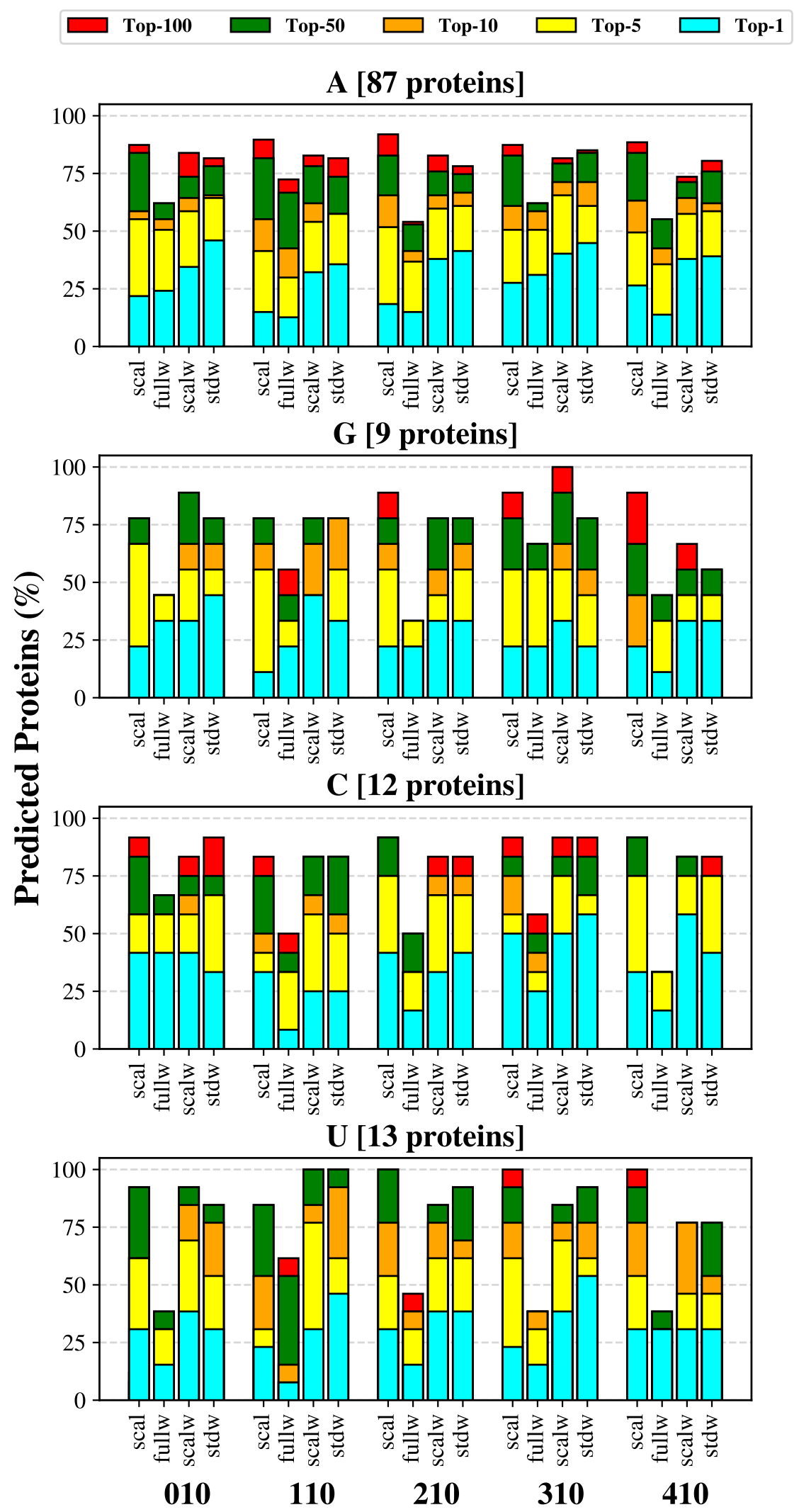

Supplementary Figure 6: Decomposition of docking powers per nucleotide type. The data are shown for the clustered distribution and each Top- $i$. 


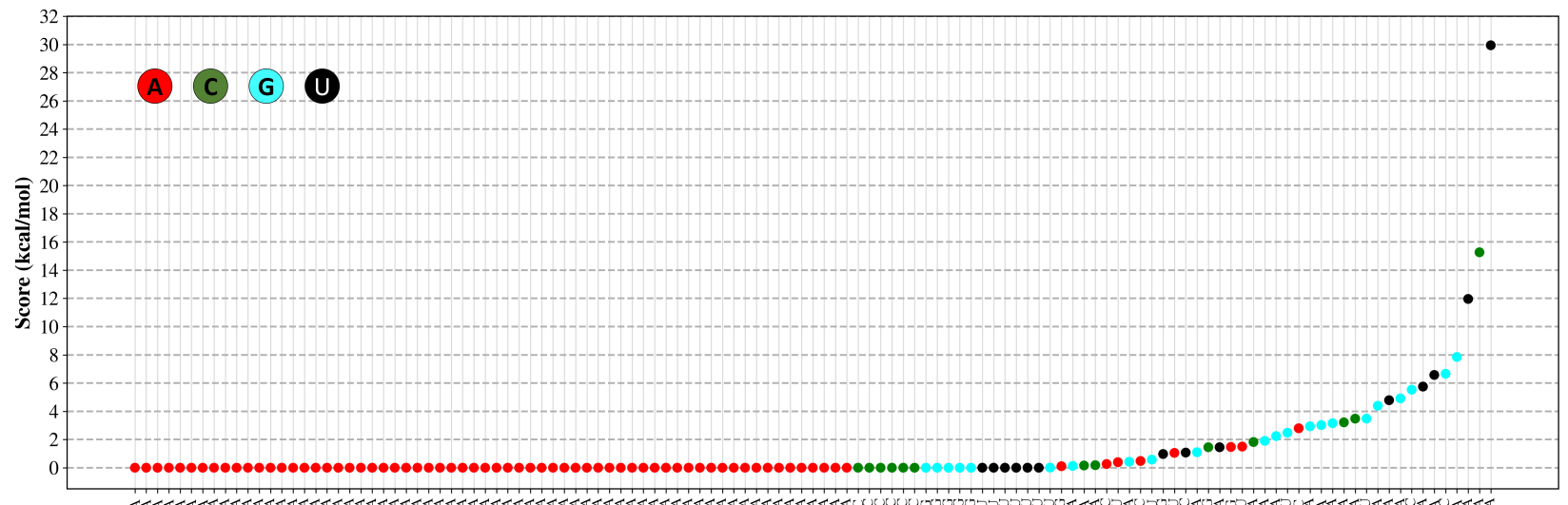

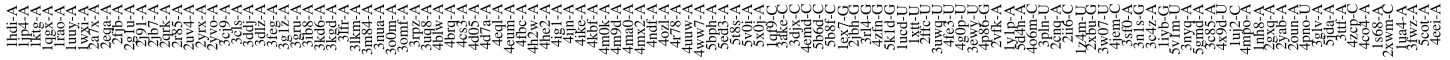

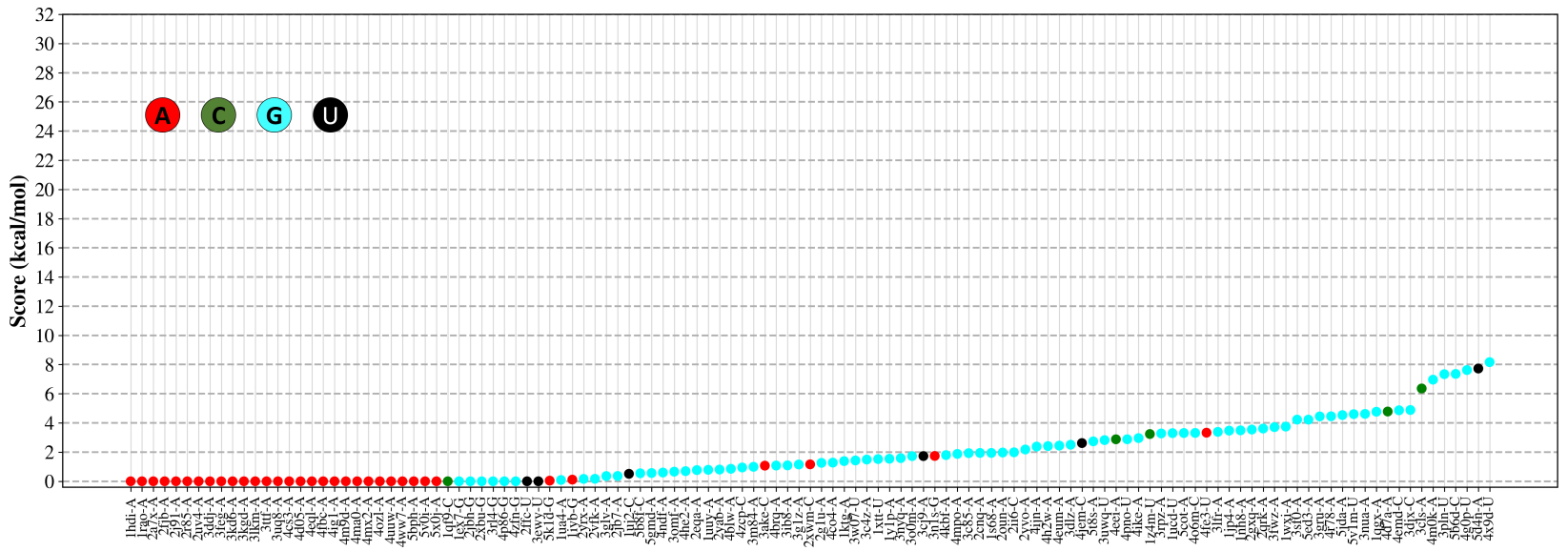

Supplementary Figure 7: Scoring differences (offset) between the best-ranked pose whatever the nucleotide type and the best-ranked pose for the nucleotide corresponding to the native ligand. Top: STDW model; bottom: SCAL model. The color code indicates the nucleotide type. 


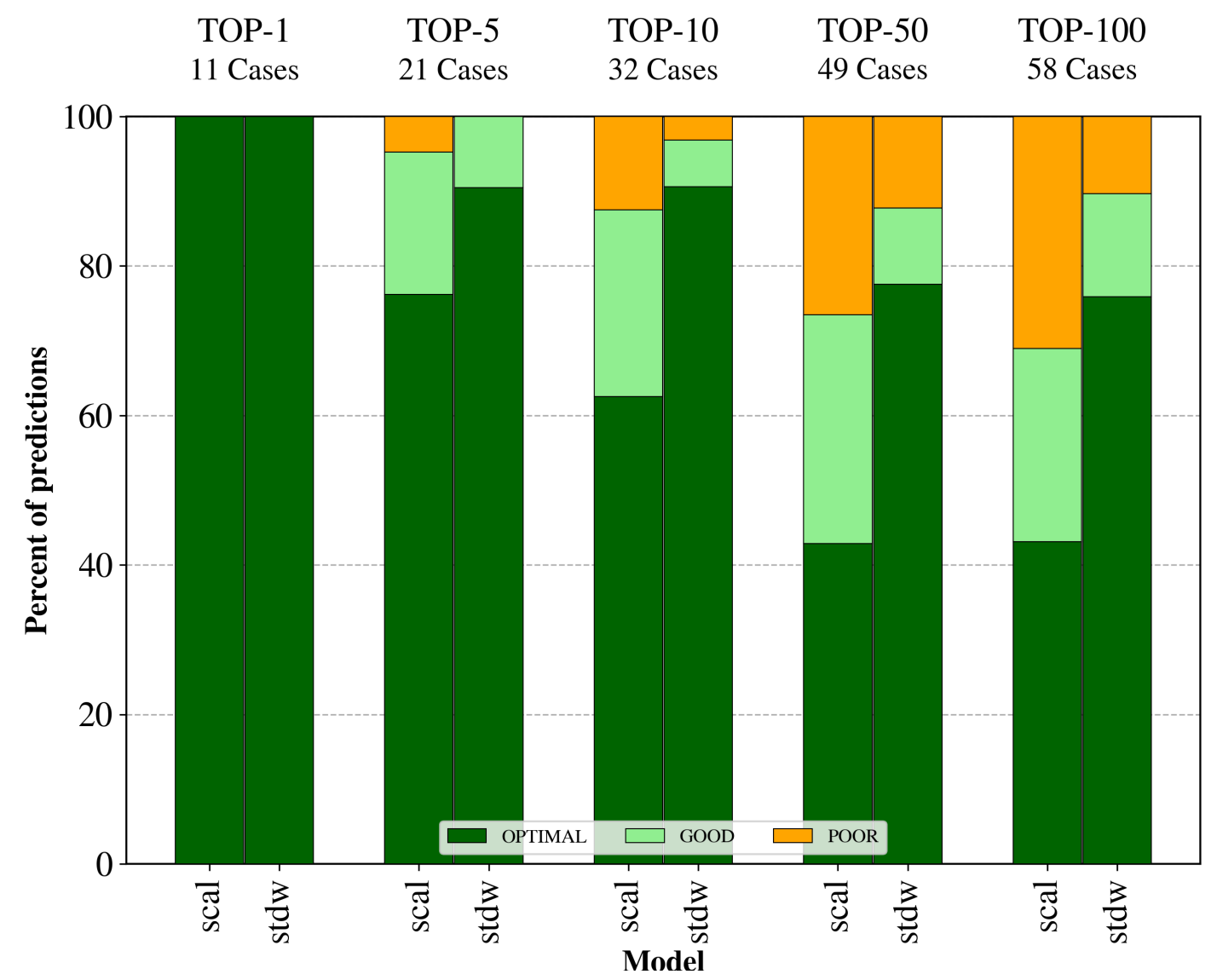

Supplementary Figure 8: Screening powers on the benchmark subset corresponding to the predictions common to the SCAL and STW models. Optimal: native nucleotide as the best ranked; good: native nucleotide in the ranked within a $2 \mathrm{kcal} / \mathrm{mol}$ range from the best ranked non-native nucleotide; poor: native nucleotide ranked out of the $2 \mathrm{kcal} / \mathrm{mol}$ range. 


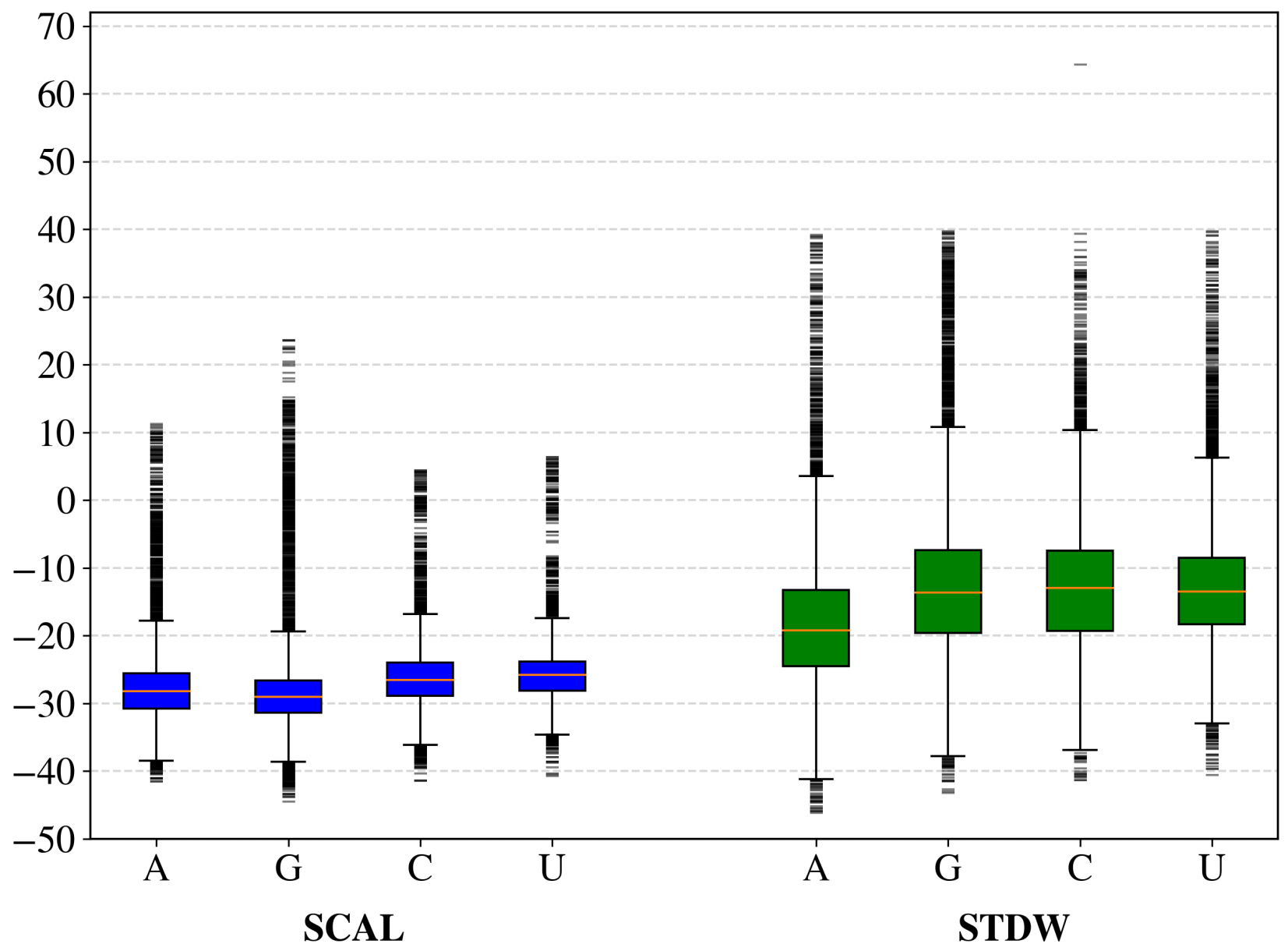

Supplementary Figure 9: Distributions of the nucleotide-dependent MCSS score for the SCAL or STDW models (R310). 


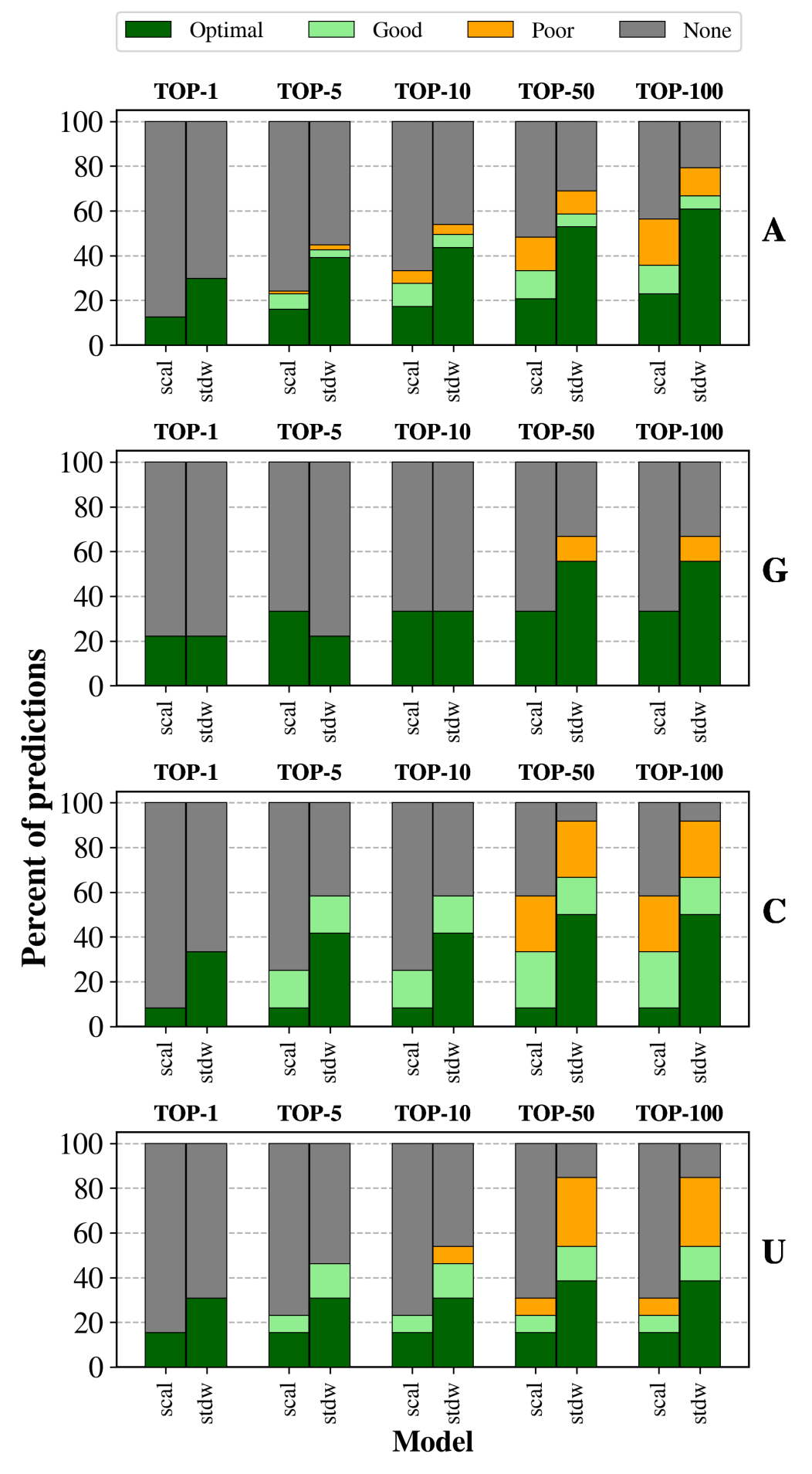

Supplementary Figure 10: Decomposition of screening powers per nucleotide type. Optimal: native nucleotide as the best ranked; good: native nucleotide in the ranked within a 2 $\mathrm{kcal} / \mathrm{mol}$ range from the best ranked non-native nucleotide; poor: native nucleotide ranked out of the $2 \mathrm{kcal} / \mathrm{mol}$ range. 


\section{Molecular features}

Supplementary Table 1: Frequencies of occurences for molecular features in the Top-10 nonpredicted cases versus benchmark. Others: presence of additional nucleotidic (nucleic acid) fragment in the binsing site; metals: presence of metal(s) in the binding site; nwat.low: presence of number of water molecules below the threshold value; vol.low: volume of the binding site below the threshold value; syn: syn conformation of the nucleic acid base; pyr: pyrimidine; pur: purine; no.base.contacts: absence of contacts with the nucleic acid base; clash_aa: clash(es) with amino-acid residues; clash_w: clash(es) with water molecules; no.salt.bridges: absence of salt-bridge; no.stacking: absence of stacking.

\begin{tabular}{|c|c|c|c|}
\hline \multicolumn{2}{|c|}{ Features } & Freq. Benchmark & Freq. no.pred \\
\hline \multirow{4}{*}{ binding site } & nwat.low & 62 & 59 \\
\hline & vol.low & 69 & 82 \\
\hline & others & 12 & 6 \\
\hline & metals & 36 & 24 \\
\hline \multirow{3}{*}{ conformational } & syn & 12 & 0 \\
\hline & pur & 79 & 71 \\
\hline & pyr & 21 & 23 \\
\hline \multirow{5}{*}{ interaction } & no.base.contacts & 12 & 12 \\
\hline & no.salt.bridges & 44 & 59 \\
\hline & no.stacking & 49 & 53 \\
\hline & clash aa & 22 & 18 \\
\hline & clash w & 33 & 41 \\
\hline
\end{tabular}


80 .

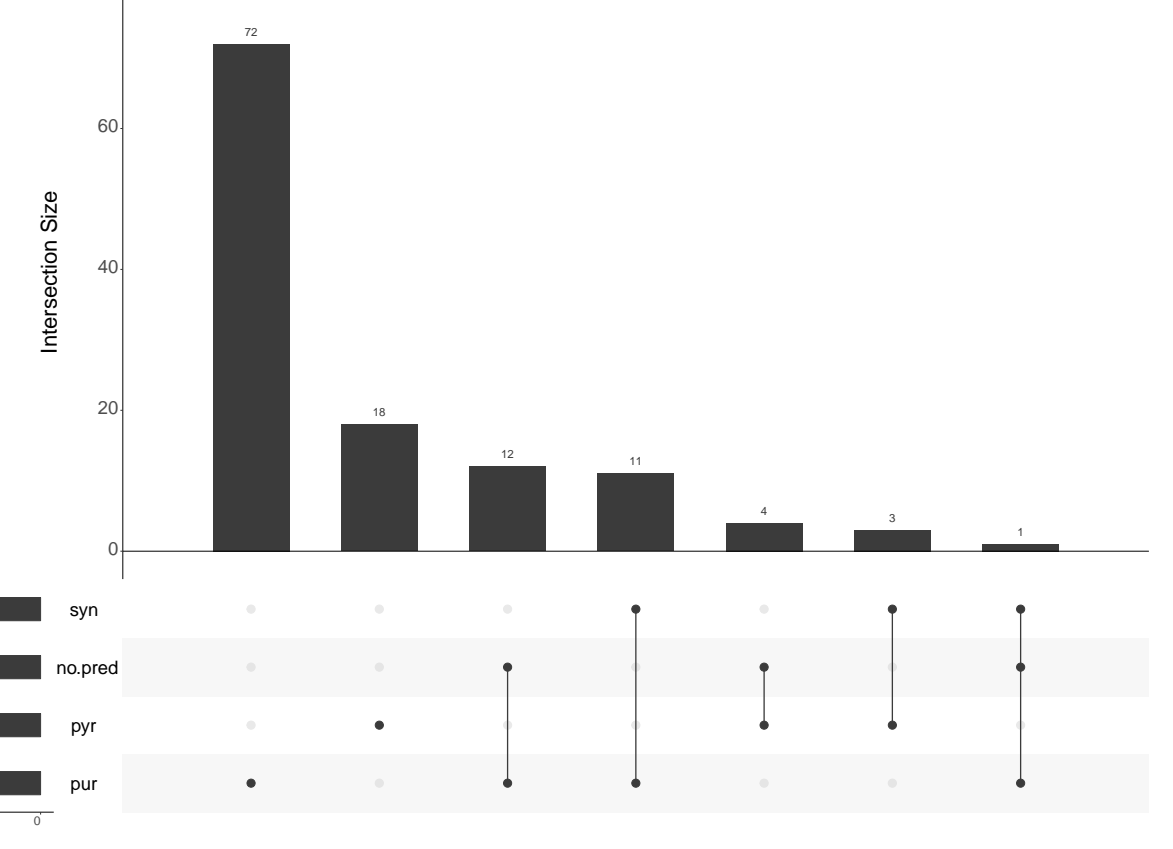

Supplementary Figure 11: Upset diagram of the impact of the conformational features on the Top-10 predictions. The intersections with only one member are not shown; syn: syn conformation of the nucleic acid base; pyr: pyrimidine; pur: purine.

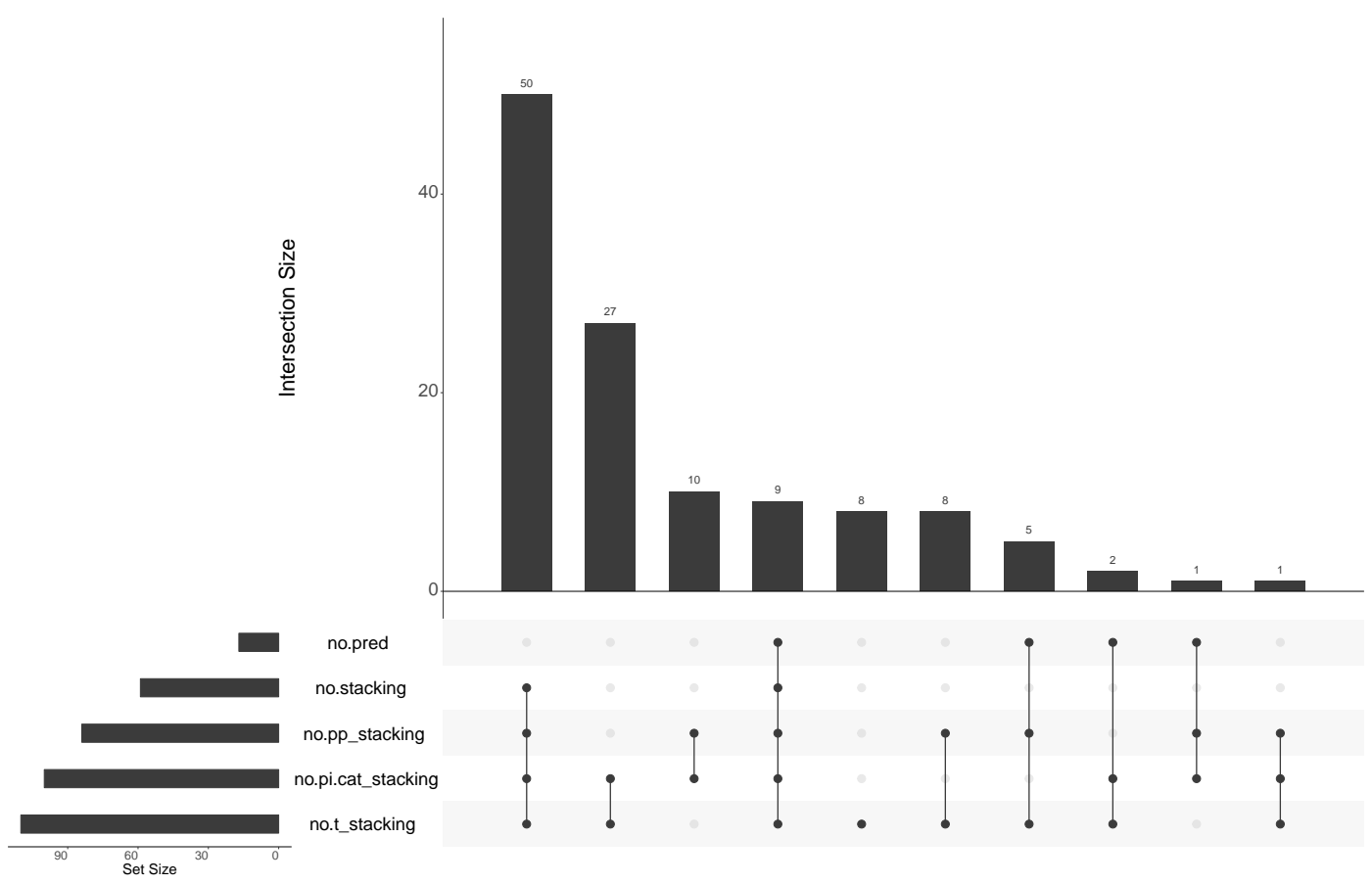

Supplementary Figure 12: Upset diagram of stacking contributions for the Top-10 predictions. no.pp_satcking: no $\pi-\pi$ stacking; no.pi.cat_stacking: no $\pi$-cation stacking; no.t_stacking: no t stacking. 
Supplementary Table 2: Frequencies of occurences for molecular features in the Top-10 for non-predicted cases of STDW-310 versus benchmark. Others: presence of additional nucleotidic (nucleic acid) fragment in the binsing site; metals: presence of metal(s) in the binding site; nwat.low: presence of number of water molecules below the threshold value; vol.low: volume of the binding site below the threshold value; syn: syn conformation of the nucleic acid base; pyr: pyrimidine; pur: purine; no.base.contacts: absence of contacts with the nucleic acid base; clash_aa: clash(es) with amino-acid residues; clash_w: clash(es) with water molecules; no.salt.bridges: absence of salt-bridge; no.stacking: absence of stacking.

\begin{tabular}{lccc}
\hline \multicolumn{2}{c}{ Features } & Freq. Benchmark & Freq. STDW(R310) \\
\hline \multirow{3}{*}{ binding site } & nwat.low & 62 & 51 \\
\cline { 2 - 4 } & vol.low & 69 & 72 \\
\cline { 2 - 4 } & others & 12 & 6 \\
\cline { 2 - 4 } & metals & 36 & 30 \\
\hline \multirow{3}{*}{ conformational } & syn & 12 & 17 \\
\cline { 2 - 4 } & pur & 79 & 83 \\
\cline { 2 - 4 } & pyr & 21 & 17 \\
\hline \multirow{3}{*}{ interaction } & no.base.contacts & 12 & 11 \\
\cline { 2 - 4 } & no.salt.bridges & 44 & 62 \\
\cline { 2 - 4 } & no.stacking & 49 & 49 \\
\cline { 2 - 4 } & clash aa & 22 & 40 \\
\cline { 2 - 4 } & clash w & 33 & 21 \\
\hline
\end{tabular}

Supplementary Table 3: Frequencies of occurences for molecular features in the Top-10 for non-optimal (good) predictions. Others: presence of additional nucleotidic (nucleic acid) fragment in the binsing site; metals: presence of metal(s) in the binding site; nwat.low: presence of number of water molecules below the threshold value; vol.low: volume of the binding site below the threshold value; syn: syn conformation of the nucleic acid base; pyr: pyrimidine; pur: purine; no.base.contacts: absence of contacts with the nucleic acid base; clash_aa: clash(es) with amino-acid residues; clash_w: clash(es) with water molecules; no.salt.bridges: absence of salt-bridge; no.stacking: absence of stacking.

\begin{tabular}{|c|c|c|c|}
\hline \multicolumn{2}{|c|}{ Features } & Freq. Benchmark & Freq. good \\
\hline \multirow{4}{*}{ binding site } & nwat.low & 62 & 60 \\
\hline & vol.low & 69 & 70 \\
\hline & others & 12 & 10 \\
\hline & metals & 36 & 60 \\
\hline \multirow{3}{*}{ conformational } & syn & 12 & 0 \\
\hline & pur & 79 & 80 \\
\hline & pyr & 21 & 0 \\
\hline \multirow{5}{*}{ interaction } & no.base.contacts & 12 & 30 \\
\hline & no.salt.bridges & 44 & 30 \\
\hline & no.stacking & 49 & 70 \\
\hline & clash aa & 22 & 20 \\
\hline & clash $\mathrm{w}$ & 33 & 40 \\
\hline
\end{tabular}


Supplementary Table 4: Variations in the binding site's volume for the subset of proteinnucleotides complexes with no prediction in the Top-10. The volume of reference corresponds to that of the experimental structure; the modified volumes are calculated for both the SCAL and STDW models. Only the cases where the variation equals or exceeds $100 \AA^{3}$ are considered. UP: increase of the binding site's volume. DOWN: decrease of the binding site's volume.

\begin{tabular}{cccc}
\hline \multicolumn{2}{c}{ Volumes } & Freq. Benchmark & Freq. nopred. \\
\hline \multirow{2}{*}{ SCAL } & UP & 12 & 0 \\
\cline { 2 - 4 } & DOWN & 19 & 18 \\
\hline \multirow{2}{*}{ STDW } & UP & 13 & 0 \\
\cline { 2 - 4 } & DOWN & 21 & 35 \\
\hline
\end{tabular}


9. Attached Supplementary Data 9 (Data-S9.txt): raw data corresponding to the number of water molecules around the ligand at a distance up to $4 \AA$.

10. Attached Supplementary Data 10 (Data-S10.csv): raw data corresponding to the variations of the binding site's volume for each protein of the benchmark in three conditions: experimental, SCAL, and STDW models.

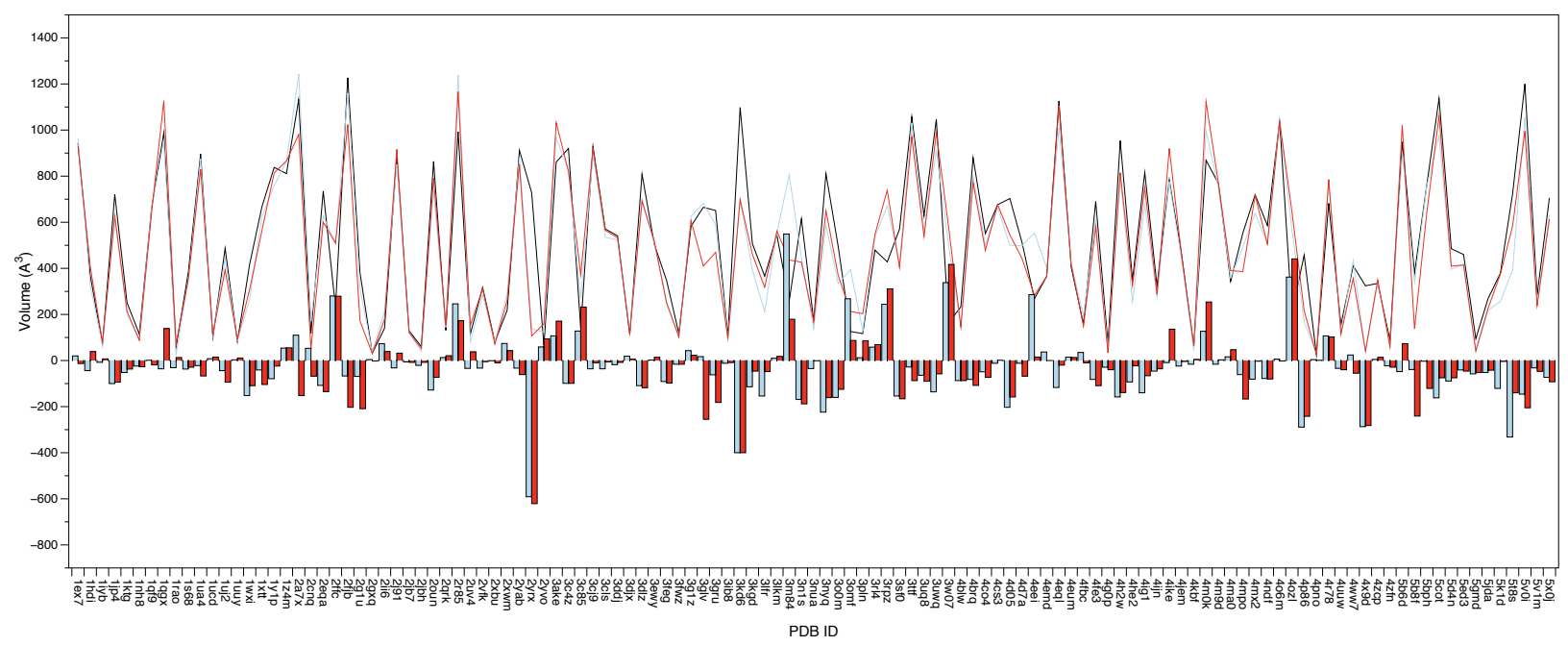

Supplementary Figure 13: Variations in the volume of the binding site. Black line: experimental structure; Blue line: optimized structure for the SCAL model; Red line: optimized structure for the STDW model. The histograms indicate a decreasing of the volume for the negative values and an increasing for the positive values. The calculation of volume does not take into account the water molecules. 
Supplementary Table 5: Impact of the nonbonded model and phosphate patch on the recovery effect of the Top-10 no-prediction subset. Y: recovered prediction using a different model and patch; N: no recovered prediction with the given model and patch.

\begin{tabular}{|c|c|c|c|}
\hline & stdw-R110 & scal-R310 & scal-R110 \\
\hline $1 \mathrm{rao}$ & $\mathrm{Y}$ & & \\
\hline $1 \mathrm{wxi}$ & $\mathrm{Y}$ & & \\
\hline $1 \mathrm{xtt}$ & $\mathrm{Y}$ & & \\
\hline $2 g 1 u$ & $\bar{Y}$ & & \\
\hline $2 \mathrm{xbu}$ & $\mathrm{Y}$ & & \\
\hline 2xwm & $\mathrm{N}$ & $\mathrm{Y}$ & \\
\hline $3 g r u$ & $\mathrm{~N}$ & $\mathrm{~N}$ & $\mathrm{~N}$ \\
\hline $3 \mathrm{~m} 84$ & $\bar{Y}$ & & \\
\hline 3nua & $\mathrm{N}$ & $\mathrm{N}$ & $\mathrm{Y}$ \\
\hline 3omf & $\mathrm{Y}$ & & \\
\hline $3 \mathrm{sf0}$ & $\mathrm{N}$ & $\mathrm{Y}$ & \\
\hline 4 eei & $\mathrm{N}$ & $\mathrm{Y}$ & \\
\hline $4 i j n$ & $\mathrm{~N}$ & $\mathrm{Y}$ & \\
\hline $4 \mathrm{zfn}$ & $\mathrm{Y}$ & & \\
\hline $5 e d 3$ & $\mathrm{Y}$ & & \\
\hline $5 \mathrm{jda}$ & $\mathrm{N}$ & $\mathrm{Y}$ & \\
\hline $5 \mathrm{v} 0 \mathrm{i}$ & $\mathrm{N}$ & $\mathrm{N}$ & $\mathrm{N}$ \\
\hline
\end{tabular}




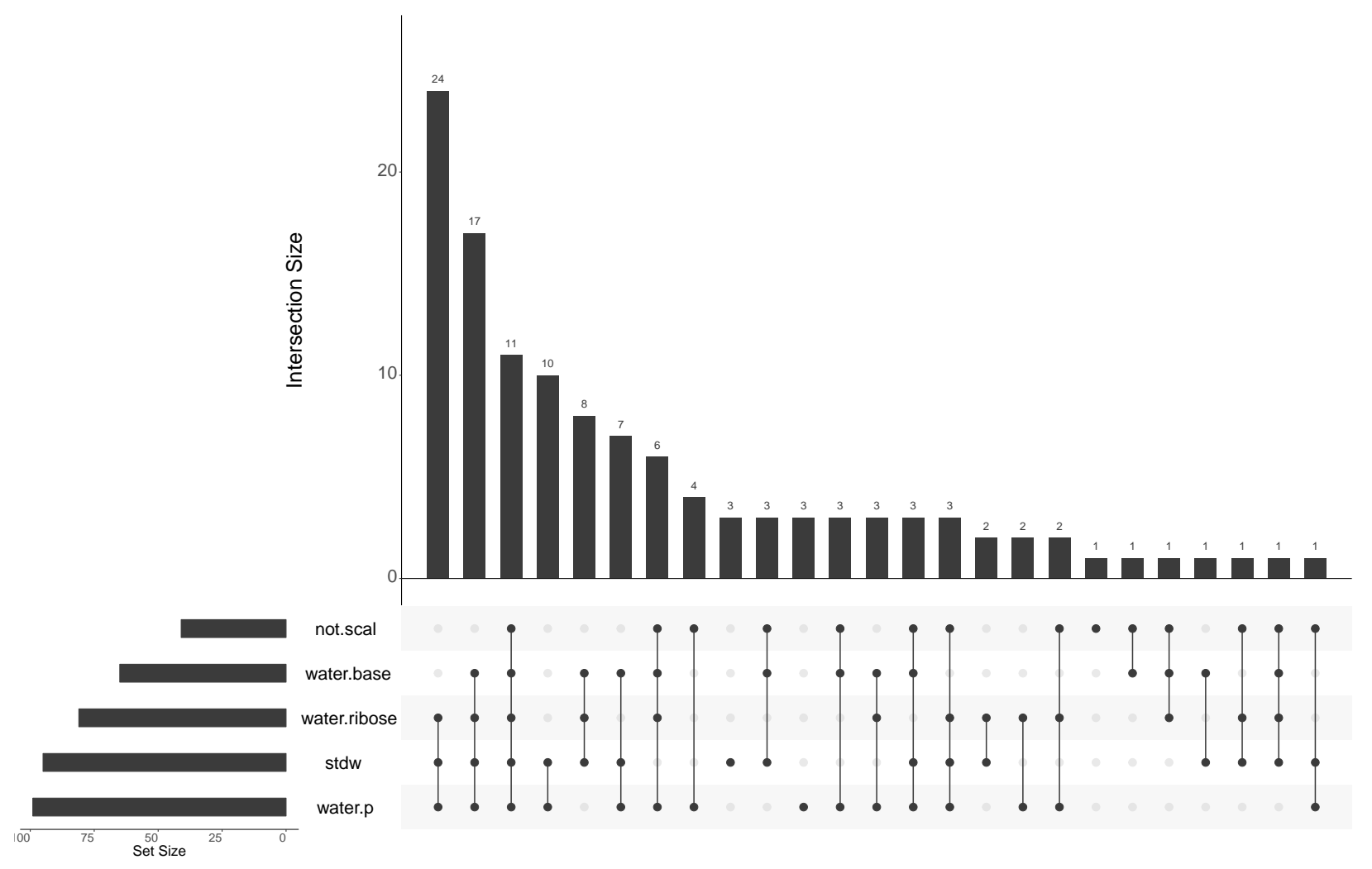

Supplementary Figure 14: Upset diagram of water-mediated contacts for the Top-10 predictions. not.scal: no prediction with the SCAL model; stdw: predictions with STDW model; water.base: presence of water-mediated contacts with the nucleic acid base; water.ribose: presence of water-mediated contacts with the ribose; water.p presence of water-mediated contacts with the phosphate group.

11. Attached Supplementary Data 11 (Data-S11.csv): raw data corresponding to the molecular features associated with the Top-10 predictions. 


\section{References}

(1) Durrant, J. D.; McCammon, J. A. BINANA: a novel algorithm for ligand-binding characterization. Journal of Molecular Graphics and Modelling 2011, 29, 888-893.

(2) OEDepict Toolkit 2.4.4.5, OpenEye Scientific Software, Santa Fe, NM.

(3) Leclerc, F.; Karplus, M. MCSS-based predictions of RNA binding sites. Theoretical Chemistry Accounts: Theory, Computation, and Modeling (Theoretica Chimica Acta) 1999, 101, 131-137.

(4) Gaillard, T. Evaluation of AutoDock and AutoDock Vina on the CASF-2013 Benchmark. Journal Of Chemical Information And Modeling 2018, 58, 1697-1706.

(5) Quiroga, R.; Villarreal, M. A. Vinardo: A Scoring Function Based on Autodock Vina Improves Scoring, Docking, and Virtual Screening. PLOS ONE 2016, 11, e0155183-18.

(6) Huang, N.; Shoichet, B. K.; Irwin, J. J. Benchmarking sets for molecular docking. Journal Of Medicinal Chemistry 2006, 49, 6789-6801.

(7) Huang, S.-Y.; Zou, X. A knowledge-based scoring function for protein-RNA interactions derived from a statistical mechanics-based iterative method. Nucleic Acids Research 2014, 42, e55-e55.

(8) Wang, C.; Zhang, Y. Improving scoring-docking-screening powers of protein-ligand scoring functions using random forest. Journal of Computational Chemistry 2017, 38, 169177.

(9) Trott, O.; Olson, A. J. AutoDock Vina: improving the speed and accuracy of docking with a new scoring function, efficient optimization, and multithreading. Journal of Computational Chemistry 2010, 31, 455-461. 